\title{
Study of Spatio-Temporal Immunofluorescence on Bead Patterns in a Microfluidic Channel
}

\author{
Venkataragavalu Sivagnanam $^{\mathrm{a}}$, Hui Yang ${ }^{\mathrm{a}, *}$ and Martin A. M. Gijs ${ }^{\mathrm{a}}$ \\ Laboratory of Microsystems, Ecole Polytechnique Fédérale de Lausanne (EPFL), 1015 Lausanne, Switzerland \\ *E-mail: hui.yang@epfl.ch
}

\begin{abstract}
We performed a direct immunoassay inside a microfluidic channel on patterned streptavidin-coated beads, which captured fluorescently-labeled biotin target molecules from a continuous flow. We arranged the beads in a dot array at the bottom of the channel and demonstrated their position- and flow rate-dependent fluorescence. As the target analyte gets gradually depleted from the flow when passing downstream the channel, the highest fluorescence intensity was observed on the most upstream positioned dot patterns. We propose a simple analytical convection model to explain this spatio-temporal fluorescence.
\end{abstract}

Keywords: Microfluidics, Bead-based immunoassay, Convection model, Magnetic bead, Diffusion. PACS: $87.15 . \mathrm{Vv}$

\section{INTRODUCTION}

Over the last two decades, tremendous progress has been made in the development of microfluidics-based biosensors [1-2]. Immunoreactions, taking advantage of the specific binding of a receptor to its target analyte, form the basis for the specific and accurate detection of analytes in a broad variety of clinical, medical, biotechnological, and environmental applications [3-4]. Both homogeneous and heterogeneous assays have been adapted to the microchip format [5-7]. A heterogeneous immunoassay exploits the interaction between a target analyte (antigen $(\mathrm{Ag})$ ) and a receptor (capture antibody $(\mathrm{Ab})$ ) immobilized on a solid phase to produce a visual, fluorescent or electrical signal, quantifying the target concentration [8]. While a continuous flow microfluidics-based bioassay allows studying the $\mathrm{Ag}$ - $\mathrm{Ab}$ binding kinetics, the intrinsically fast $\mathrm{Ag}-\mathrm{Ab}$ reaction rate can be mass transport-limited [9-12]. In this case, the Ag in the sample flow can get depleted, resulting in a non-uniform exposure of the Ag to the available binding surface. We recently reported a method, based on electrostatic self-assembly, for realizing an onchip immunoassay using streptavidin-coated beads as assay substrate for the detection and quantification of target $\mathrm{Ag}$ [13]. Bead immobilization occurred through electrostatic force between the negatively-charged bead surface and a positively-charged aminosilane micro-pattern applied to a glass substrate [13-14]. The receptor Ab was coupled to the bead surface for specific binding with the target Ag. In this paper we report a microfluidic assay performed on streptavidin-coated bead patterns and show a spatio-temporal dependent analyte capture, by measuring the fluorescence signal on the dot patterns along the microchannel. We propose a simple analytical convection model to explain the analyte distribution and corresponding fluorescent signal.

\section{EXPERIMENTAL DETAILS}

The direct immunoassay was performed in a microfluidic chip, which was fabricated by reversibly bonding a poly-dimethylsiloxane (PDMS) replica containing the microfluidic channel with a height of $20 \mu \mathrm{m}$, a width of 100 $\mu \mathrm{m}$ and a length of $20 \mathrm{~mm}$ to a glass substrate having (3-aminopropyl) triethoxysilane (APTES) dot patterns. Forming the substrate of the immunoassay, $1.05 \mu \mathrm{m}$ streptavidin-coated beads were self-assembled on the APTES dot templates due to electrostatic force. The distance between two dots in a column spanning the microfluidic channel cross-section was $15 \mu \mathrm{m}$. The distance between two such consecutive columns in the flow direction was also $15 \mu \mathrm{m}$, while the dots in two consecutive columns were each time shifted by $7.5 \mu \mathrm{m}$. Thereafter, a washing step

CP1311, $8^{\text {th }}$ International Conference on the Scientific and Clinical Applications of Magnetic Carriers edited by U. Häfeli, W. Schütt, and M. Zborowski

(C) 2010 American Institute of Physics 978-0-7354-0866-1/10/\$30.00 
was done by flowing a PBS solution through the microfluidic channel using a neMESYS syringe pump (Cetoni, Korbussen, Germany). Fig. 1(a) schematically illustrates the in situ micropatterns of the streptavidin-coated beads. After the bead micropatterning process, the microchannel walls were blocked using $1 \times$ PBS-BSA (1\%) solution for $\sim 2$ min. This step is essential to avoid the unspecific adsorption of Abs or Ags on the microchannel walls. Following this, Atto488-labeled biotin (Sigma-Aldrich, Buchs, Switzerland) was chosen as analyte to be captured on the bead surface, due to its strong binding kinetics and fast diffusion rate. The experiment was performed by flowing $500 \mathrm{~nL}$ of $100 \mathrm{pg} / \mathrm{mL}$ of biotin solution through the microchannel at flow rates of $0.25 \mathrm{~nL} / \mathrm{s}$ and $1 \mathrm{~nL} / \mathrm{s}$, respectively. The different steps of the immunoassay protocol are illustrated in Fig. 1(b), indicating (i) the APTES patterns, (ii) the streptavidin-coated beads, and (iii) the fluorescently-labeled biotin molecules captured on the bead surface. The final washing was performed by flowing a PBS-Tween (0.5\%) (PBST) solution for 2 min.
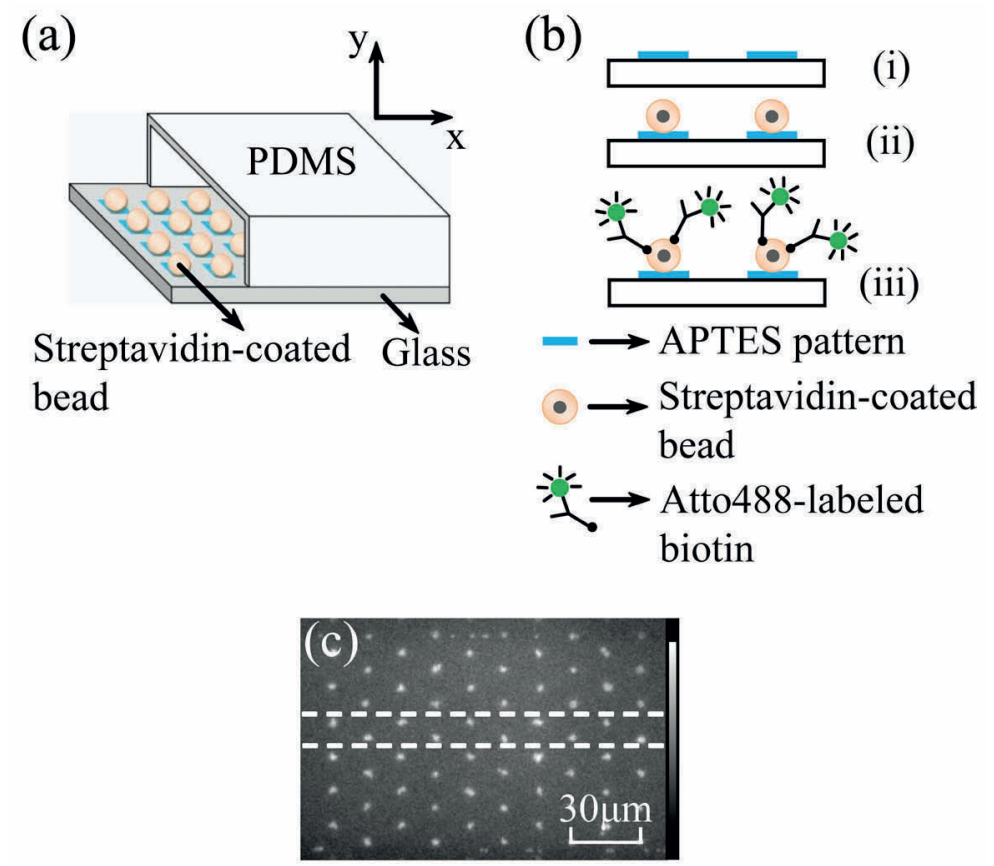

FIGURE 1. (a) Schematic illustration of the streptavidin-coated beads in situ patterned on an APTES template inside a microfluidic channel. (b) Schematic illustration of the direct immunoassay on the streptavidin-coated bead patterns. (c) Fluorescence micrograph after performing the direct immunoassay, showing columns of subsequent dot patterns. The fluorescence signal intensity is recorded along a rectangular zone in the flow direction indicated by the white dashed lines.

For fluorescence detection and image acquisition of the immunoassay, we used an ORCA-C4742-95ER CCD camera (Hamamatsu, Hamamatsu, Japan) mounted on an inverted microscope Axiovert S100 (Zeiss), with a 40× objective (LD Achroplan, numerical aperture 0.5). The microscope was equipped with a X-Cite ${ }^{\circledR} 120$ mercury short arc lamp module (EXFO, Mississauga, Canada) and the filter set for Atto488 dye. The exposure time used for capturing the fluorescence signal was 2 seconds, and AquaCosmos software (Hamamatsu) was used for the fluorescence image analysis. Fig. 1(c) shows a representative fluorescence micrograph of ten consecutive columns of bead patterns after the direct immunoassay. In the experiment, the fluorescence signal is recorded along a long narrow rectangle, which passes over subsequent dots in the microfluidic channel. Signals from up to 150 consequent dots in total were obtained. Due to the experimental configuration, the bead patterns in the more upstream positioned columns will acquire a stronger fluorescence signal compared to those patterns downstream, which means that the target analyte molecules, by diffusion, are gradually depleted from the flow when passing over subsequent dot columns.

Fig. 2 presents the fluorescence intensity profile recorded along 80 subsequent bead dot patterns after performing the direct immunoassay at a flow rate of $0.25 \mathrm{~nL} / \mathrm{s}$. The first peak and the last peak correspond to a single dot pattern in the first and the 80th column downstream the flow. The fluorescence intensity observed from the upstream dot patterns is higher than the downstream ones, which means that the immuno-fluorescence signal obtained after continuous flow sample exposure has a spatio-temporal distribution. 


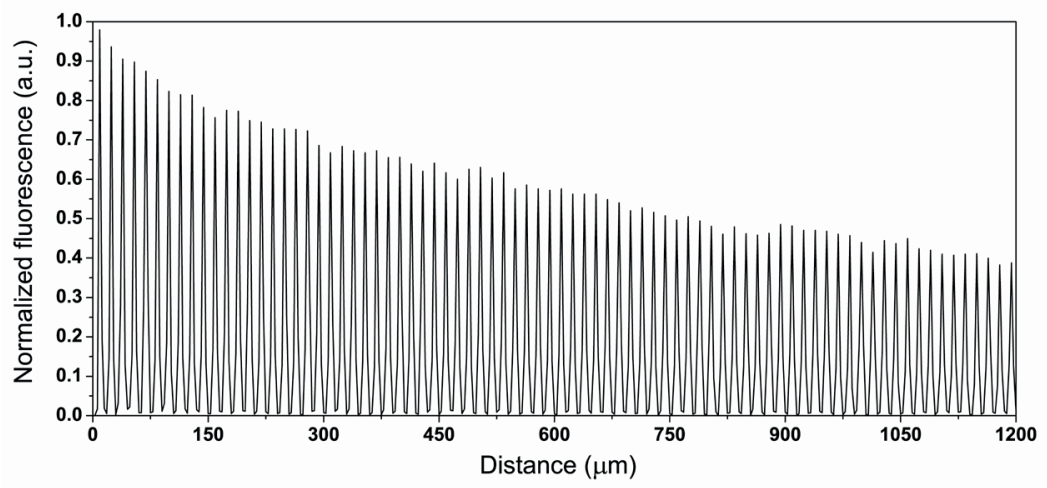

FIGURE 2. Fluorescence intensity profile along 80 subsequent bead dot patterns after performing the direct immunoassay at a flow rate of $0.25 \mathrm{~nL} / \mathrm{s}$. The first peak and the last peak correspond to the single dot pattern of the first and the $80^{\text {th }}$ column downstream the flow.

\section{MATHEMATICAL MODEL}

To explain our experimental results, we developed a convection model in two-dimensions, which is a reasonable approximation, since the variations in Ag concentration across the width of microchannel can be neglected (model for an infinitely wide channel). Fig. 3 schematically illustrates the microfluidic channel with the patterned magnetic beads. The analyte solution flows into the microfluidic channel at a constant average flow rate. At low Reynolds number conditions (Reynolds number $<<1$ ), the analyte flow inside the microchannel is typically laminar and has a parabolic flow profile. The analyte molecules are transported by advection along the flow (x-direction) and are free to diffuse in the y-direction. We assume that all target analytes, once diffused to the bottom surface of the channel are captured by one of the bead patterns (i.e., a 100\% capture efficiency when they come in contact with the bead surface). We position the origin of the coordinate system at the bottom of the channel, $7.5 \mu \mathrm{m}$ upstream relative to the first column of dot patterns.

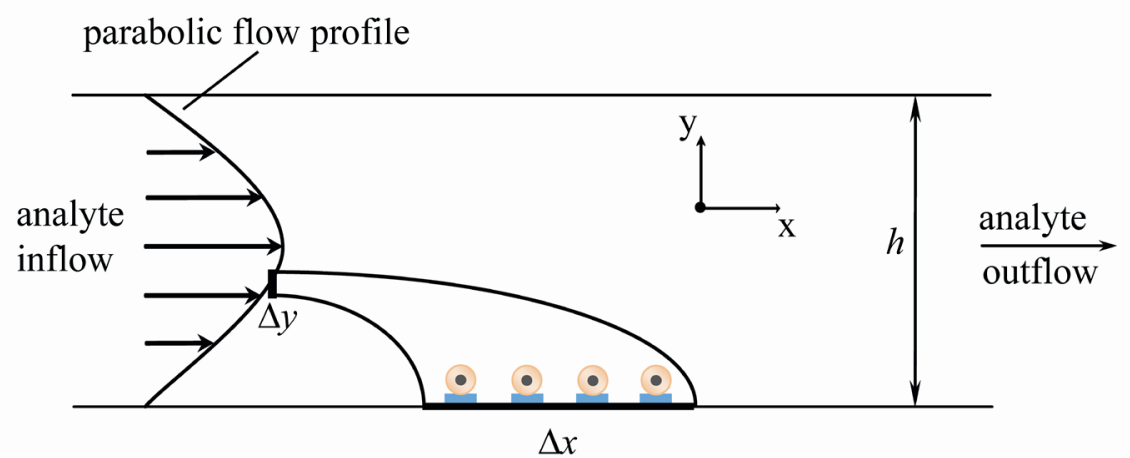

FIGURE 3. Schematic of the flow conditions for performing the direct immunoassay in a microfluidic channel of height $h$. The analyte solution flows into the channel from the left inlet and has a parabolic flow profile. The analyte molecules entering over a distance $\Delta y$ are transported in the channel and, after diffusion to the bottom of the channel, are captured by the streptavidincoated bead patterns situated in an interval $\Delta x$.

The number of analyte molecules that enter the microchannel and are distributed over an infinitesimal distance $\Delta y$ is $v(y) t C_{m} \Delta y$ where $C_{m}$ is the molecular concentration per unit length, $v(y)$ is the flow rate at position $y$ and time $t$. When the number of target molecules captured on the dot patterns distributed over an infinitesimal distance $\Delta x$ is defined as $\varphi(x) t \Delta x$, in which $\varphi(x)$ is a function expressing the molecular distribution along the flow direction, we obtain $\varphi(x) \Delta x=C_{m} v(y) \Delta y$ for high-affinity binding and when capture sites on the beads are non-saturated. When using pressure-driven flow, the velocity profile is parabolic: 


$$
v(y)=\frac{3}{2} v_{c}\left[1-\left(\frac{y-h / 2}{h / 2}\right)^{2}\right]
$$

where $h$ is the height of the microfluidic channel and $v_{c}$ is the average velocity $(125 \mu \mathrm{m} / \mathrm{s}$ and $500 \mu \mathrm{m} / \mathrm{s}$ for the 0.25 $\mathrm{nL} / \mathrm{s}$ and $1 \mathrm{~nL} / \mathrm{s}$ flow rates, respectively). Assuming that the transport in the $y$ direction is driven only by diffusion, results in the following simple relation [15]:

$$
y=(D t)^{1 / 2}=\left(D \frac{x}{v(y)}\right)^{1 / 2}
$$

where $D$ is the diffusion coefficient. Substituting Eq. 1 into Eq. 2 and then calculating the derivative $d y / d x$, results in an expression for $\Delta y / \Delta x$. Thereafter, substituting this result into the relation $\varphi(x) \Delta x=C_{m} v(y) \Delta y$, we obtain:

$$
\varphi(x)=\frac{2 C_{m} D}{h}\left[1-\frac{1}{h}\left(\frac{2 D x}{3 v_{c}}\right)^{1 / 2}\right]
$$

from which we derived the normalized function $f(x)$ :

$$
f(x) \equiv \varphi(x) \frac{h}{2 C_{m} D}=1-\frac{1}{h} \sqrt{\frac{2 D x}{3 v_{c}}}
$$

\section{RESULTS AND DISCUSSION}

The analytical result shown in Eq. 4 allows the study of the influence of flow rate and analyte diffusion coefficient on the analyte distribution and corresponding fluorescent signal. In this section, the proposed model is applied to evaluate the effect of these two important parameters on the immunofluorescence. Unless mentioned differently, the parameters used are as follows: the height of the microfluidic channel $h=20 \mu \mathrm{m}$, the diffusion coefficient of the analyte molecule $D=2.2 \times 10^{-11} \mathrm{~m}^{2} / \mathrm{s}$ for the calculations with the flow speed as parameter, and the average flow speed of the analyte solution $v_{c}=125 \mu \mathrm{m} / \mathrm{s}$ for the calculation for the calculations with the diffusion coefficient as parameter.

Fig. 4(a) shows the predicted fluorescence distribution at different flow conditions. In this figure, the fluorescent signal is represented as a function of the distance from the beginning of the bead pattern area and decreases along the flow direction, which indicates that the analyte gets gradually depleted from the flow when passing over subsequent columns of dot patterns. Moreover, this depletion increases with decreasing flow velocity. For an average flow velocity of $125 \mu \mathrm{m} / \mathrm{s}$, corresponding to a flow rate of $0.25 \mathrm{~nL} / \mathrm{s}$ for the channel used in the experiments, the fluorescent signal decreases to about $20 \%$ of the initial intensity, which is much more pronounced compared to the decrease to $70 \%$ obtained for the flow rate of $1 \mathrm{~nL} / \mathrm{s}$ (average velocity of $500 \mu \mathrm{m} / \mathrm{s}$ ). Therefore, if the number of sample molecules is limited (small volume or low concentration sample), a slow flow velocity should preferably be used. Furthermore, the fluorescence distribution is affected by the value of the analyte's diffusion coefficient, as shown in Fig. 4(b). For the molecules with $D=2.2 \times 10^{-11} \mathrm{~m}^{2} / \mathrm{s}$, the fluorescent signal decreases to about $20 \%$, which is approximately four times less than the value obtained for analyte molecules with a diffusion coefficient $D=1 \times 10^{-12} \mathrm{~m}^{2} / \mathrm{s}$.

In order to verify the validity of the proposed model, we compare the results from the experiment with the predictions of the model. In particular, Fig. 5 presents the fluorescence intensity profile recorded along 150 subsequent bead dot patterns after performing the direct immunoassay at a flow rate of $1 \mathrm{~nL} / \mathrm{s}$ (grey plot) and 0.25 $\mathrm{nL} / \mathrm{s}$ (black plot), respectively. The first peak and the last peak correspond to a single dot pattern in the first and the 150th column downstream the flow. Each dashed curve in Fig. 5 represents a fit using Eq. 4 with a diffusion coefficient $D=2.2 \times 10^{-11} \mathrm{~m}^{2} / \mathrm{s}$, in the range of earlier reported values. Indeed, Hu et al. [10] studied the reaction kinetics of antigen-antibody binding in electrokinetically controlled microfluidic heterogeneous immunoassays 
using a numerical method, taking an antigen diffusion coefficient of $D=1.5 \times 10^{-11} \mathrm{~m}^{2} / \mathrm{s}$ in their computational model. Kamholz et al. [16] proposed an optical measurement method for quantitative analysis of molecular diffusion in a Tshaped microchannel. Their experiments showed a diffusion coefficient that was dependent on flow rate; when the flow rate was less than $50 \mathrm{~nL} / \mathrm{s}$, corresponding to an average flow velocity smaller than $2 \mathrm{~mm} / \mathrm{s}$, a diffusion coefficient less than $2.5 \times 10^{-10} \mathrm{~m}^{2} / \mathrm{s}$ was found for a fluorescein-biotin complex. Moreover, in the work of Buranda et al. [17], typical diffusion coefficients for biomolecules of the order of $\leq 10^{-11} \mathrm{~m}^{2} / \mathrm{s}$ were mentioned.

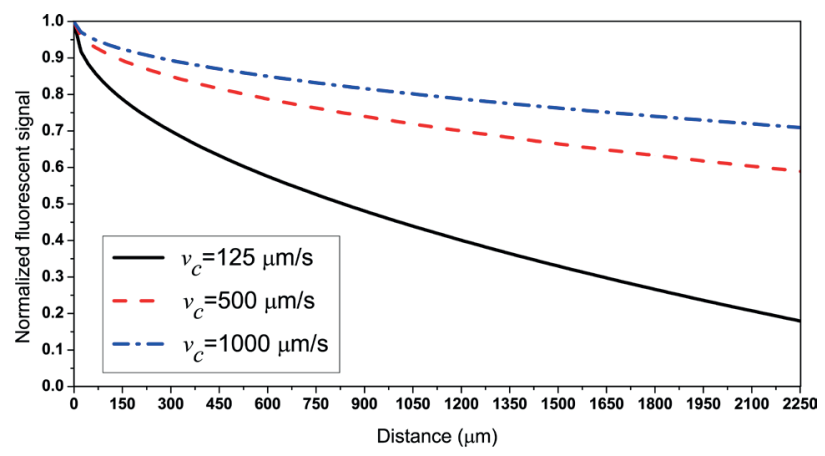

(a)

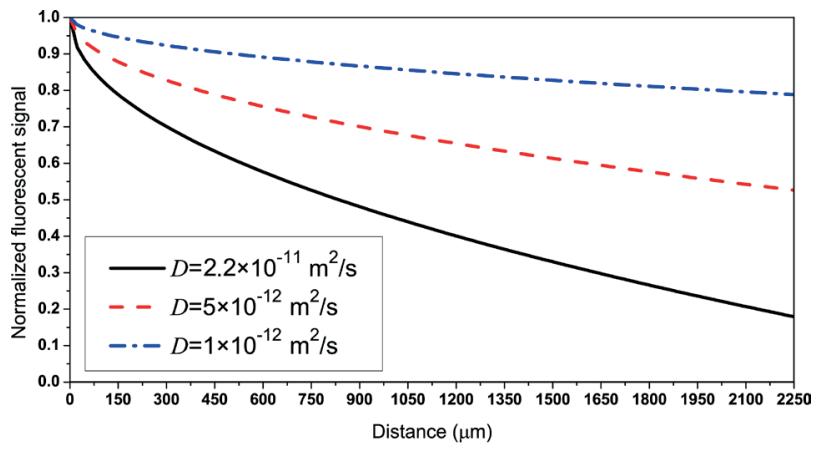

(b)

FIGURE 4. Calculated normalized fluorescent signal in the $x$-direction (a) for molecules with a diffusion coefficient $D=2.3 \times 10^{-11} \mathrm{~m}^{2} / \mathrm{s}$ and flow velocities of 125,500 and $1000 \mu \mathrm{m} / \mathrm{s}$, respectively, and (b) for molecules with an average flow velocity $v_{c}=125 \mu \mathrm{m} / \mathrm{s}$ and diffusion coefficients of $1 \times 10^{-12}, 5 \times 10^{-12}$ and $2.2 \times 10^{-11} \mathrm{~m}^{2} / \mathrm{s}$, respectively.

(a)

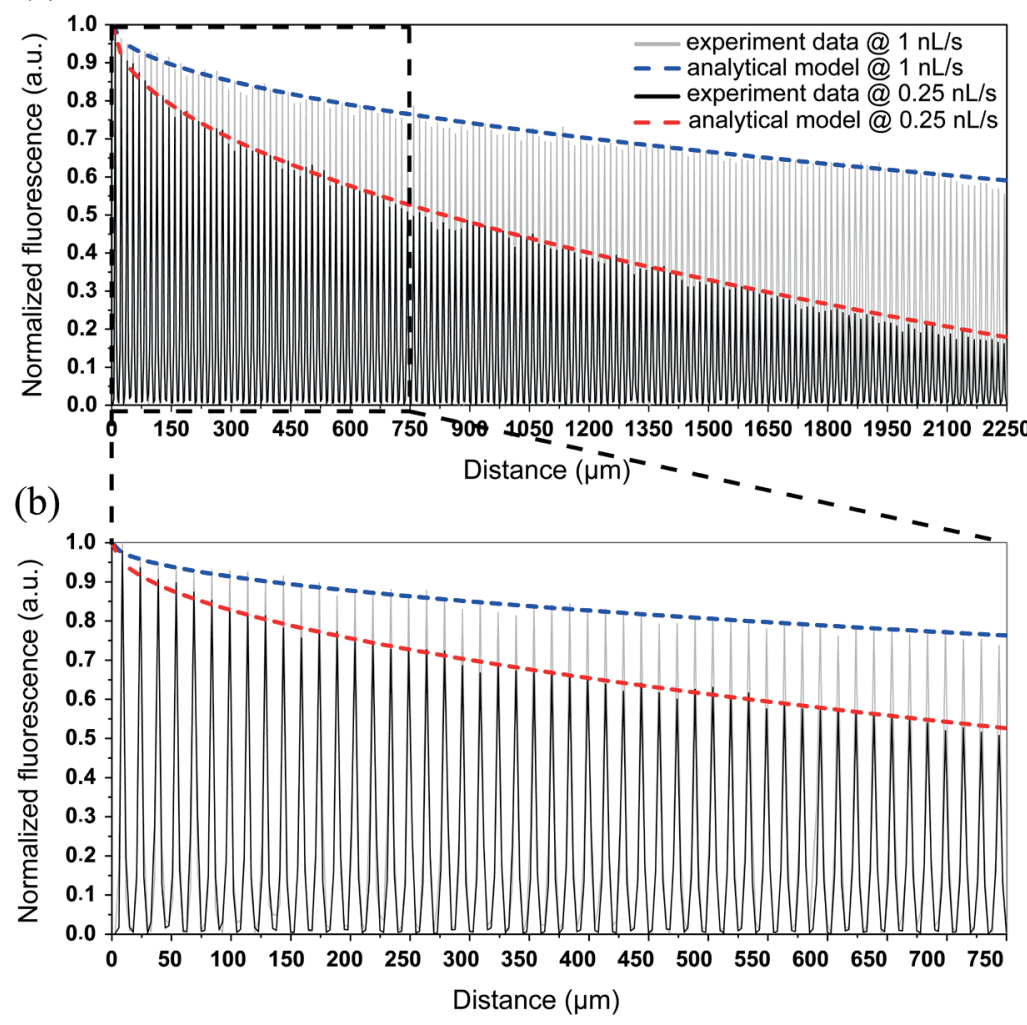

FIGURE 5. (a) Fluorescence intensity profile along 150 subsequent bead dot patterns after performing the direct immunoassay at a flow rate of $1 \mathrm{~nL} / \mathrm{s}$ (grey plot) and $0.25 \mathrm{~nL} / \mathrm{s}$ (black plot), respectively. The first peak and the last peak correspond to the single dot pattern of the first and the $150^{\text {th }}$ column downstream the flow. Simulation results from our analytical convection model are shown as the dotted curves. (b) Detail of the fluorescence intensity of the first 50 subsequent bead dot patterns. 


\section{CONCLUSION}

In conclusion, we have studied both experimentally and theoretically the spatio-temporal immunofluorescence of biotin target analyte molecules that are specifically captured from a continuous sample flow. This direct immunoassay is completely performed on-chip using streptavidin-coated beads as assay substrate. The highest fluorescence intensity corresponds to the dot pattern which is most upstream positioned in the flow. The analytical modeling indicates that the target analyte by convection gets gradually depleted from the flow, when the latter passes over subsequent dot columns. The fluorescence intensity profile is consistent with a square root functional decrease as a function of the position of the dots and the inverse flow rate. We think, our approach holds much promise for application as a method that yields quickly, by a one-time observation of the fluorescence, information about the dynamics of the analyte-bead interaction process and on the space-time-dependence of the developing fluorescent signal.

\section{ACKNOWLEDGMENTS}

The authors are grateful to the Swiss National Science Foundation (200020-121558) for funding of this project.

\section{REFERENCES}

1. D. R. Reyes, D. Iossifidis, P. A. Auroux and A. Manz, Anal. Chem. 74, 2623-2636 (2002).

2. A. P. F. Turner, Science 290, 1315-1317 (2000).

3. R. P. Ekins, J. Chem. Educ. 76, 769-780 (1999).

4. C. P. Price and D. J. Newman, Principles and Practice of Immunoassay, London: Macmillan, 1997.

5. N. Chiem and D. J. Harrison, Clin. Chem. 44, 591-598 (1998).

6. E. Delamarche, A. Bernard, H. Schmid, B. Michel and H. Biebuyck, Science 276, 779-781 (1997).

7. A. Dodge, K. Fluri, E. Verpoorte and N. F. de Rooij, Anal. Chem. 73, 3400-3409 (2001).

8. A. Bange, H. B. Halsall and W. R. Heineman, Biosens. Bioelectron. 20, 2488-2503 (2005).

9. I. Chaiken, S. Rose and R. Karlsson, Anal. Biochem. 201, 197-210 (1992).

10. G. Hu, Y. Gao and D. Li, Biosens. Bioelectron. 22, 1403-1409 (2007).

11. M. Zimmermann, E. Delamarche, M. Wolf and P. Hunziker, Biomed. Microdevices 7, 99-110 (2005).

12. M. A. M. Gijs, F. Lacharme and U. Lehmann, Chem. Rev. 110, 1518-1563 (2010).

13. V. Sivagnanam, B. Song, C. Vandevyver and M. A. M. Gijs, Anal. Chem. 81, 6509-6515 (2009).

14. V. Sivagnanam, A. Sayah, C. Vandevyver and M. A. M. Gijs, Sens. Actuators B 132, 361-367 (2008).

15. J. Lagowski, A. M. Kontkiewicz, L. Jastrzebski and P. Edelman, Appl. Phys. Lett. 63, 2902-2904 (1993).

16. A. E. Kamholz, E. A. Schilling and P. Yager, Biophys. J. 80, 1967-1972 (2000).

17. T. Buranda, J. Huang, V. H. Perez-Luna, B.Schreyer, L. A. Sklar and G. P. Lopez, Anal. Chem. 74, 1149-1156 (2002). 